\title{
Magnetic Fields in Blazar Pc-scale Jets - Possible connection to Spin Rates of Black holes ?
}

\author{
P. Kharb ${ }^{1}$ \\ ${ }^{1}$ Dept. of Physics, Purdue University, IN 47907, USA. E-mail: pkharb@physics.purdue.edu \\ M. L. Lister ${ }^{1}$, and P. Shastri ${ }^{2}$ \\ ${ }^{2}$ Indian Institute of Astrophysics, Bangalore, India.
}

\begin{abstract}
We re-examine the differences observed in the pc-scale magnetic field geometry of high and low optical polarization Quasars (HPQs, LPRQs) using the MOJAVE sample. We find that, as previously reported, HPQ jets exhibit predominantly transverse $B$ fields while LPRQ jets tend to display longitudinal $B$ fields. We attempt to understand these results along with the different $B$ field geometry observed in the low and high energy peaked BL Lacs (LBLs, HBLs) using a simple picture wherein the spinning central black holes in these AGNs influence the speed and strength of the jet components (spine, sheath). Higher spin rates in HPQs compared to LPRQs and LBLs compared to HBLs could explain the different total radio powers, VLBI jet speeds, and the observed $B$ field geometry in these AGN classes.
\end{abstract}

\section{Introduction}

The relativistically beamed active galactic nuclei (AGNs) fall primarily in two categories on the basis of their total radio power and optical spectra - the radio powerful Quasars exhibit strong, broad and narrow emission lines, while the relatively lower radio power BL Lacertae objects display weak or no emission-lines. In the radio-loud Unified Scheme, FRII and FRI radio galaxies are considered to be the parent population of Quasars and BL Lacs, respectively [1].

Quasars and BL Lacs however, do not form a homogeneous class with similar properties. Low optical polarization radio Quasars (LPRQs) consistently reveal core optical fractional polarization, $m_{\text {opt }} \leq 3 \%$, while the high optical polarization Quasars (HPQs) routinely reveal highly polarized optical cores with $m_{\text {opt }} \geq 3 \%$ [2]. The BL Lacs likewise seem to fall under two subclasses. The basis for the division is however their spectral energy distributions (SEDs) the synchrotron peaks of the low-energy peaked BL Lacs (LBLs) lie in the near-IR/optical regime, while they lie in the UV/soft X-ray regime for the highenergy peaked BL Lacs (HBLs).

The Quasar and BL Lac subclasses display additional systematic differences. The HPQs and the LBLs seem to exhibit "extreme" behaviour in their respective classes - they show greater variability, greater radio core power, greater radio core prominence and larger misalignments between their pcand kpc-scale radio jets compared to LPRQs and HBLs, respectively ( [3] and references in [4]). It has been proposed that these differences are on account of greater Doppler beaming in their cores/jets due to being oriented at smaller angles to our line of sight. Furthermore, Very Long Baseline Interferometry polarization (VLBP) observations of the beamed AGNs have revealed that while the majority of HPQ jets display transverse magnetic $(B)$ fields, LPRQ jets display mostly longitudinal $B$ fields [3]. While LBLs predominantly display transverse $B$ fields in their pcscale jets [5], HBL jets tend to exhibit longitudinal $B$ fields [4]. Orientation alone is insufficient in producing the different $B$ field structures in Quasars and BL Lacs [3, 4].

In this paper, we re-examine the trend observed in the pc-scale $B$ field structures of Quasars using a larger sample, and attempt to understand the different properties of HPQs, LPRQs, LBLs and HBLs in the different black hole spin rate scenario.

\section{Objects of Study}

The Quasars belong to the MOJAVE (Monitoring Of Jets in AGNs with VLBA Experiments) sample [6], while the BL Lacs belong to the 1-Jy, HEAO-1 and RGB samples [4]. The classification of Quasars as HPQ/LPRQ was adopted from [3, 7]. Optical polarization data is however not yet available for all the MOJAVE sources and additional optical monitoring is necessary. The VLBP observations of the Quasars and BL Lacs were made at 15 and $5 \mathrm{GHz}$, respectively. Note that the previous observations that showed a difference in the $B$ field structures of HPQs and LPRQs were made at 22 and $43 \mathrm{GHz}$ [3]. 
Quasar jets exhibit pc-scale rotation measures of the order of $\sim 500 \mathrm{rad} \mathrm{m} \mathrm{m}^{-2}$ or less [8]. At $15 \mathrm{GHz}$ the expected Electric Vector Position Angle (EVPA) rotation is only about $10^{\circ}$. The BL Lac jets typically exhibit lower rotation measures, of the order of a few $100 \mathrm{rad} \mathrm{m}^{-2}$ or less. Assuming an RM of $\sim 100 \mathrm{rad} \mathrm{m}^{-2}$ would result in an EVPA rotation of $\sim 20^{\circ}$ at $5 \mathrm{GHz}$. We therefore do not expect significant changes in the observed $B$ field trend for Quasars, while it may have some effect on the trend observed in BL Lacs.

\section{Results}

The Quasar sample considered here consists of four times as many HPQs (37) and twice as many LPRQs (17) than were used by [3]. Using this larger sample, we find that the trend of different $B$ field structures observed previously, gains further in significance. The two-sided Kolmogorov-Smirnov (KS) test indicates that the probability $(p)$ of the HPQ and LPRQ EVPA data being drawn from the same distribution is only $3 \%$ (Fig. 1). The probability decreases to $0.3 \%$ when only the inner jet (with projected length $r<15 \mathrm{pc}$ ) is considered.

Using the total radio luminosity at $1.4 \mathrm{GHz}$ of the HPQs and LPRQs from the NASA/IPAC Extragalactic Database (NED), we find no significant difference between the HPQ and LPRQ distributions (KS test $p=55 \%$ ). However, when we restrict the redshift range to $z<1.0$, we find that the HPQs and LPRQs differ with a probability $>90 \%$ (Fig. 2). The KS test indicated that the redshift distribution of HPQs and LPRQs was not significantly different before $(p=64 \%)$ or after making the cut in redshift $(p=20 \%)$, suggesting that the luminosity difference in HPQs and LPRQs is not a redshift effect. However, in the case of the LBLs and HBLs, although the total radio power distributions differed significantly ( $p=0.001 \%$, see [4]) we could not rule out this bias since the redshifts of the LBLs were systematically higher than the HBLs.

Total radio power, however, is affected by Doppler boosting effects in the core. We are currently reducing Very Large Array (VLA) $1.4 \mathrm{GHz}$ data for all the sample objects. This will allow us to directly compare the unbeamed extended emission, which will serve as a better indicator of jet power, in these sources.
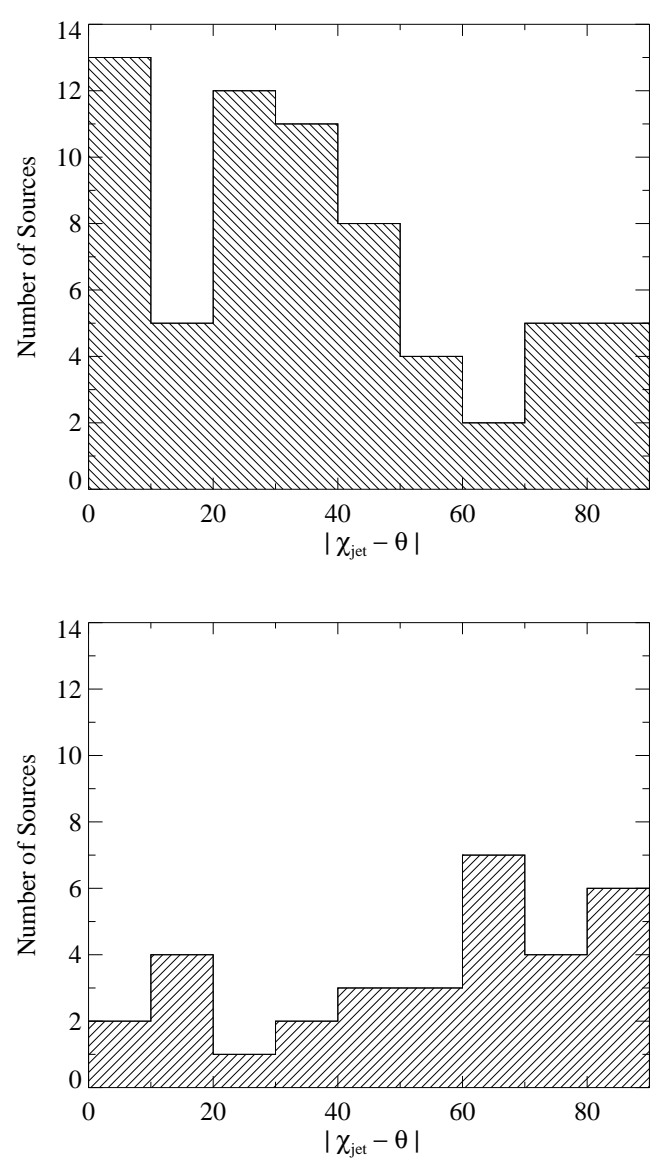

Fig. 1. The distribution of the $15 \mathrm{GHz}$ jet EVPA, $\chi_{j e t}$, w.r.t. the VLBI jet direction $\theta$ in HPQs (Top) and LPRQs (Bottom).

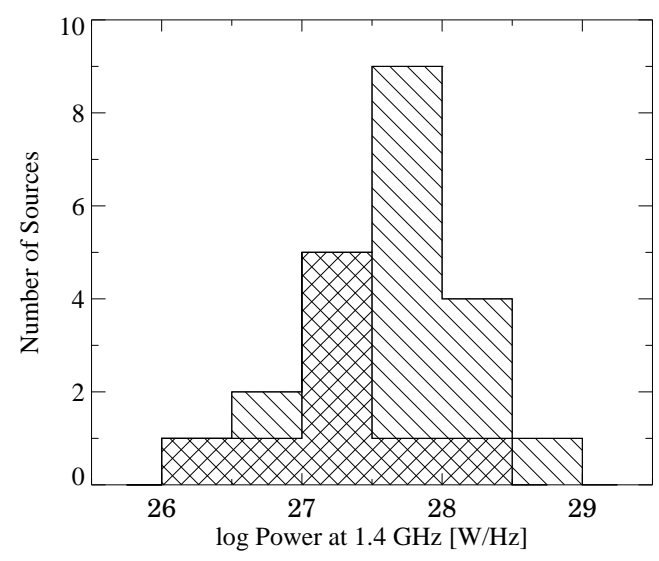

Fig. 2. The distribution of total radio luminosity at $1.4 \mathrm{GHz}$ for HPQs (shaded with stripes at $-45^{\circ}$ ) and LPRQs (shaded with stripes at $+45^{\circ}$ ) with redshifts $<1.0$. 


\subsection{Spine-sheath Jets \& Black hole Spins}

Meier [9] has demonstrated through MHD jet simulations that the jet power can be linked directly to the black hole angular momentum, so that jets with greater spins would result in jets with greater speed and power. We present here a simple picture by which the different $B$ field structures observed in HPQs, LPRQs, LBLs and HBLs, and their different total radio powers, can be reconciled. At the core of the argument lies the supposition that both FRI and FRII sources have jets with a spine-sheath structure, with the faster spine displaying predominantly transverse $B$ fields and the slower sheath displaying longitudinal $B$ fields. Such a magnetic field configuration could arise due to helical fields [10], or due to a combination of wound-up $B$ field lines in the jet center resulting from a rotating black hole-accretion disk system [11], and a shear layer resulting from jet-medium interaction [10]. A sheath could also result due to the flow acceleration being a function of the angular distance from the jet axis [15]; or on pc-scales, due to an accretion disk wind [16]. Possible spine-sheath $B$ field structures have been observed in both FRI and FRII jets on kpc [13] and pc-scales $[4,14]$.

Furthermore, we assume that the spin rates of the rotating black holes influence both the speed and strength of the spine and sheath, i.e., dictates which jet component dominates the overall emission due to Doppler boosting effects, and the width of the jet.

Assuming that HPQs and LBLs are intrinsically more powerful than LPRQs and HBLs, respectively (Fig. 2 and [4]), we can interpret the $B$ field geometry in the scenario of different black hole spin rates. In this picture, the spin rates decrease from the HPQs to LPRQs, and from LBLs to HBLs. This is illustrated in Fig. 3 for a jet with a spine-sheath structure on pc-scales. Both the spine and sheath contribute to the overall $B$ field morphology, consistent with the observed EVPAs ranging from $0^{\circ}$ to $90^{\circ}$. However, the spine dominates in the highest spin rate HPQs, while lower spin rates in LPRQs result in a less dominant spine. At small angles to line of sight this would result in predominantly transverse $B$ fields in HPQs and longitudinal (sheath-dominated) $B$ fields in LPRQs. Relatively smaller angles to line of sight are required for HPQs to the extent that the narrow spine is not missed. Due to the lower spin rates in BL Lacs, the spine would be weaker and broader. HBLs may not have a prominent spine at all, resulting in largely longitudinal $B$ fields. A weak spine but larger angles to line of sight in HBLs would also be consistent with the VLBP observations. It is also possible that the spine is present only on $\mu$ arcsec-scales, as required by the $\mathrm{TeV}$ data [17].

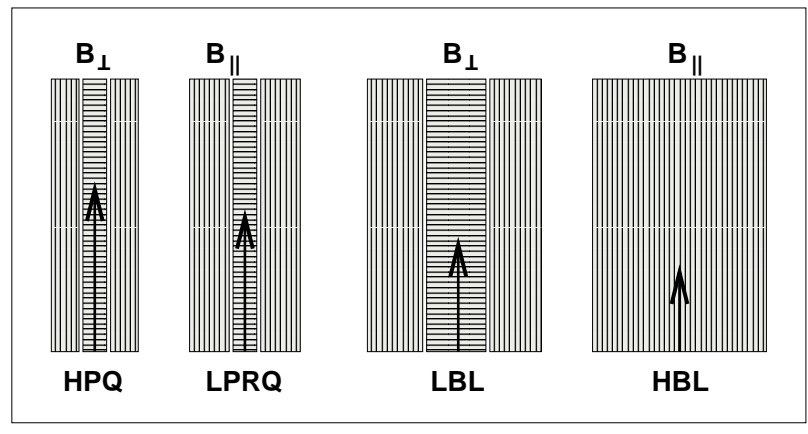

Fig. 3. Illustration of a spine-sheath jet structure on pcscales close to the central engine. The spine and sheath are shaded with horizontal and vertical stripes, respectively, to suggest the dominant transverse and longitudinal $B$ fields (note that HBLs could still have a weak spine, see text). Spin rates decrease from HPQs to LPRQs and from LBLs to HBLs. This influences the jet speed and power, indicated by the length of the arrows.

\subsection{Jet Speeds, Radio core power $\mathcal{G}$ Misalignment}

We find that the apparent speeds of HPQ jets are systematically higher than in the LPRQs (KS test $p>90 \%$, Fig. 4; Lister et al., 2008, in prep.), while LBL jets seem to be faster than HBL jets $(p>99.8 \%$, [4]), consistent with the different spin rate model.

As radio cores are the unresolved bases of radio jets, higher radio core powers in the HPQs and LBLs compared to LPRQs and HBLs, respectively, are consistent with more powerful radio jets, due to higher black hole spins, in these objects.

Misalignments between pc- and kpc-scale jets can be used as a statistical indicator of jet orientation [18]. However, misalignment measurements may be influenced by the spatial resolution and the dynamic range of the radio images used, and must be treated with caution. If HPQs and LBLs indeed have larger misalignments between their pc and $\mathrm{kpc}$-scale jets than LPRQs and HBLs, respectively, then we could understand that as being due to broader jets 

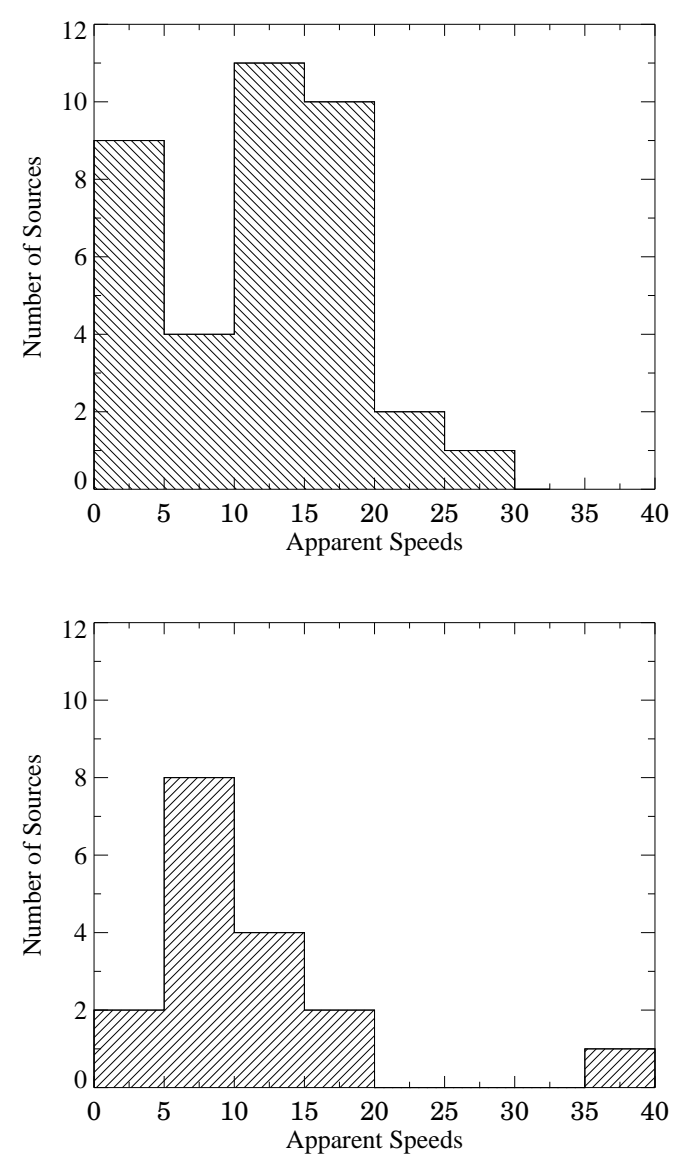

Fig. 4. The distribution of jet apparent speeds in HPQs (Top) and LPRQs (Bottom).

in LPRQs and HBLs (see [19] who suggest larger jet opening angles in HBLs). In broader outflows, small bends in the jet may not be as apparent, leading to seemingly straighter jets and smaller misalignments. However we note that the misalignment issue is somewhat contentious, with different studies reporting different results [4].

\subsection{Caveats \& Testing the Model}

The simple model that we have proposed here could not be the complete picture however. Pc-scale spinesheath jet structures have not been observed in the majority of Quasars or BL Lacs. Although this could be related to the sensitivity of the VLBP observations, a more complex jet structure is highly probable. Different black hole spin rates alone cannot account for the different emission-line strengths in Quasars and BL Lacs. Differences in the nuclear ISM of these AGNs are likely. This in turn will influence the spine-sheath structure due to jet interaction. Differences in the broad-band SEDs of BL Lacs have also been suggested to arise due to differences in accretion rates [21]. A way to test the model would be to examine if the HPQ jets are indeed the most collimated and narrow, while the HBL jets are the least collimated and the broadest. Moreover, assuming that black hole spins dictate the jet directional stability, misalignments if measurable, should progressively increase from HPQs to HBLs.

\section{Conclusions}

We have attempted to understand the different inferred magnetic field structures in Quasar and BL Lac subclasses in a simple model wherein the black hole spin rates influence the speed and strength of the jet components (spine, sheath), thereby producing either predominantly transverse (dominant spine) or longitudinal (weak spine, dominant sheath) $B$ fields. In this model the black hole spin rates decrease from HPQs to LPRQs and from LBLs to HBLs. This model is consistent with the lower total radio power, weaker radio cores and the lower apparent VLBI jet speeds in LPRQs and HBLs, compared to HPQs and LBLs, respectively. An evolutionary scenario in which LPRQs evolve from HPQs and HBLs from LBLs, is consistent with the proposed model.

\section{References}

[1] C. Urry and P. Padovani, PASP 107, 803 (1995)

[2] J. Angel and H. Stockman, ARAA 18, 321 (1980)

[3] M. Lister and P. Smith, ApJ 541, 66 (2000)

[4] P. Kharb et al., MNRAS 384, 230 (2007)

[5] D. Gabuzda et al., MNRAS 319, 1109 (2000)

[6] M. Lister and D. Homan, $A J$ 130, 1389 (2005)

[7] C. Impey et al., ApJ 375, 46 (1991)

[8] R. Zavala and G. Taylor, ApJ 612, 749 (2004)

[9] D. Meier, ApJ 522, 753 (1999)

[10] R. Laing, ApJ 248, 87 (1981)

[11] D. Meier, et al., Science 291, 84 (2001)

[12] R. Laing, ASP Conf. Ser. 100: Energy Transport in Radio Galaxies and Quasars 100, 241 (1996)

[13] M. Hardcastle et al., MNRAS 278, 273 (1996)

[14] J. Attridge et al., ApJL 518, L87 (1999)

[15] G. Ghisellini et al., A $\& A$ 432, 401 (2005)

[16] R. Blandford and A. Levinson, ApJ 441, 79 (1995)

[17] B. Piner and P. Edwards, ApJ 600, 115 (2004)

[18] V. Kapahi and D. Saikia, JApA 3, 465 (1982)

[19] P. Wiita et al., ArXiv e-prints , 0707.3456 (2007)

[20] M. Orr and I. Browne, MNRAS 200, 1067 (1982)

[21] M. Böttcher and C. Dermer, ApJ 564, 86 (2002) 